\title{
Innovación educativa en el grado de Educación Social de las universidades españolas: una revisión sistemática
}

\author{
Silvia Martínez de Miguel López \\ Juan Antonio Salmerón Aroca \\ Pedro Moreno Abellán \\ Universidad de Murcia. España. \\ silviana@um.es \\ jasa2@um.es \\ pma8@um.es
}

Recibido: 31/5/2019

Aceptado: 3/2/2020

Publicado: 20/7/2020

\section{Resumen}

Desde finales del siglo xx se ha venido produciendo en Europa un importante cambio ideológico a la hora de entender la formación universitaria a través de múltiples dimensiones. Uno de los principales cambios afectaba a los procesos metodológicos y evaluativos desarrollados por los docentes de la educación superior. Este artículo aborda el perfil de las innovaciones didácticas que se han ido incorporando en el espacio universitario utilizando como contexto el grado de Educación Social en las distintas universidades españolas que lo tienen en su catálogo de títulos. Para ello, la metodología empleada fue la revisión sistemática. Partiendo de un $N=510$ registros publicados en abierto en los últimos 10 años en bases de datos como Web of Science, Scopus o Eric, se seleccionaron finalmente 38 investigaciones. Los hallazgos reflejan que se está produciendo un cambio de metodologías, recursos e instrumentos de evaluación docente, mayoritariamente en referencia al empleo de recursos tecnológicos por parte del profesorado, además de contribuir al aprendizaje autónomo, significativo, participativo y dialógico del alumnado.

Palabras clave: enseñanza universitaria; innovación educativa; educación social

Resum. Innovació educativa en el grau d'Educació Social de les universitats espanyoles: una revisió sistemàtica

Des de finals de segle xx s'ha produït a Europa un important canvi ideològic a l'hora d'entendre la formació universitària a través de múltiples dimensions. Un dels principals canvis afectava els processos metodològics i avaluatius desenvolupats pels docents de l'educació superior. Aquest article aborda el perfil de les innovacions didàctiques que s'han anat incorporant a l'espai universitari utilitzant com a context el grau d'Educació Social a les diferents universitats espanyoles que l'han incorporat al seu catàleg de títols. Per fer-ho, la metodologia emprada ha estat la revisió sistemàtica. Partint d'un $N=510$ registres publicats en obert en els últims 10 anys en bases de dades com ara Web of Science, Scopus o Eric, finalment se'n van seleccionar 38 investigacions. Les troballes reflecteixen que s'està produint un canvi de metodologies, recursos i instruments d'avaluació docent, majorità- 
riament en referència a la utilització de recursos tecnològics per part del professorat, a més de contribuir a l'aprenentatge autònom, significatiu, participatiu i dialògic de l'alumnat.

Paraules clau: ensenyament universitari; innovació educativa; educació social

Abstract. Educational innovation in social education bachelor's degrees in Spanish universities: A systematic review

From the end of the twentieth century, an important ideological change influenced our understanding of multiple dimensions of higher education training in Europe. One of the principal changes affected the methodological and evaluative processes of university students. This article examines the types of educational innovations that have been incorporated in the higher education setting, particularly in bachelor's programmes in social education in the Spanish universities that offer this degree. The methodology used was systematic review. Based on 510 records published in the last 10 years in databases such as the Web of Science, Scopus or Eric, 38 investigations were finally selected. The findings reflect that there has been a change in the methodologies, resources and instruments used for evaluation, mostly regarding teachers' use of technological resources, but also other contributions to achieve autonomous, meaningful, participatory and dialogic learning by students.

Keywords: higher education teaching; educational innovation; social education

\section{Sumario}
1. Introducción y estado de la cuestión
4. Conclusiones
2. Material y método
Referencias bibliográficas

3. Resultados y discusión

\section{Introducción y estado de la cuestión}

La Declaración de Bolonia en el año 1999 provocó toda una revolución en el sistema universitario, puesto que ocasionó importantes cambios en la estructuración de la formación en dicho nivel educativo. Una de las grandes trasformaciones está relacionada con el cambio de paradigma metodológico y de concepción de lo que supone el proceso de enseñanza-aprendizaje universitario. Con la creación del Espacio Europeo de Enseñanza Superior se introducen nuevas metodologías y estrategias de actuación que otorgan un mayor protagonismo a los estudiantes frente a las corrientes tradicionales de los estudios superiores, que quedaban focalizados en el predominio de la clase magistral como estrategia metodológica.

De acuerdo con Delgado y De Justo (2018), este nuevo paradigma sitúa al alumnado como el centro del proceso de enseñanza-aprendizaje, porque asienta el supuesto de que un aprendizaje eficaz solo es posible si aquel es responsable de organizar y desarrollar su trabajo. Por ello es importante con- 
templar diferentes estrategias y recursos que puedan conducir a favorecer una mayor implicación y protagonismo de los estudiantes.

En el escenario universitario europeo existe un común denominador a la hora de contemplar la heterogeneidad de estrategias docentes para fomentar la actividad y un mayor grado de implicación del alumnado a partir de modelos formativos más abiertos y flexibles (De Pablos, 2007). Desde estas premisas irrumpe con fuerza el concepto de innovación educativa en la enseñanza universitaria, que ofrece una pluralidad de estrategias que promueven el desarrollo de actividades formativas caracterizadas por la creatividad y la flexibilidad, las cuales, sin duda, motivan al alumnado en su proceso de aprendizaje (Martínez de Miguel, 2007). Las principales dimensiones que se señalan para acotar este concepto de innovación educativa de la mano de especialistas como García Peñalvo (2015) serían: actividad; tecnología; métodos y técnicas, y resultados.

En este trabajo se pretende ampliar la perspectiva desde la consideración de la innovación como estrategia de mejora de la acción docente, sea cual sea el recurso utilizado. De acuerdo con Matas, Tójar y Serrano (2004, p. 1): «se trata, por tanto, de una acción planificada que implica la existencia de un cambio no madurativo, evolutivo o casual, sino un cambio perseguido». Y en ese sentido, como bien afirma Michavila (2009), los profesores son esenciales para la incorporación de propuestas de mejora docente, las cuales van a estar condicionadas por su motivación y formación. Con ello se convierten en actores decisivos en cuanto a su complicidad y protagonismo para desarrollar los procesos de cambio educativo, así como las innovaciones pertinentes. En ese sentido representó un choque frontal con la mentalidad tradicional de lo que suponía había de ser la enseñanza superior y todo un complejo proceso de sensibilización, motivación y formación al que el profesorado universitario tuvo que enfrentarse superando muchos obstáculos en el camino, ya que conllevaba un giro de 180 grados en la cultura universitaria. Como muy bien expresa Díaz (2010, p. 2):

La innovación de las prácticas educativas y del currículo, en lo que atañe a la participación de los profesores, sólo será posible en la medida en que el docente se desenvuelva en una cultura profesional basada en el pensamiento estratégico y participe en una comunidad de discurso crítico orientada a la transformación de la docencia. Es decir, lo que se necesita es construir una cultura de la innovación que descanse en el desarrollo de comunidades de aprendizaje profesional entre el profesorado.

En ese sentido, y como afirma Sirignano (2017), hoy en día no puede concebirse la universidad si no es como una entidad que tiene entre sus objetivos el aprendizaje a lo largo de todo el ciclo vital. Por ello la innovación no tiene que convertirse en un imperativo institucional, sino más bien que, a través de un ejercicio de reflexión profesional crítico acerca de la práctica docente, asentado paulatinamente en la importancia del intercambio y el coaprendizaje, se pueden introducir mejoras sustanciales que den respuesta a las necesidades de 
la formación universitaria actual. No obstante, a pesar de las dificultades del cambio de paradigma en dicho escenario, desde la implantación de los primeros grados en España en el curso académico 2008-2009 se han ido incorporando acciones de mejora respecto a esta perspectiva.

En el caso específico del grado de Educación Social el reto además era relevante. Se trataba de una formación universitaria con cierta «juventud», que asistía a una mejora en cuanto a su propia estructura y contenido curricular, así como a su asentamiento profesional en la sociedad, que se equiparaba a formaciones con mayor experiencia y reconocimiento en el ámbito investigador y de evaluación de las propias competencias profesionales. Pero una formación que llevaba implícita la adquisición de competencias fundamentales por las que los futuros profesionales en el ámbito de la educación social condujeran a la formación de ciudadanos reflexivos, críticos, conscientes de los cambios que acaecen en la realidad, así como comprometidos por la transformación y la mejora social colectiva. En ese sentido, muchas de las estrategias metodológicas de innovación que se pueden incorporar en las aulas universitarias podrían favorecer dicho planteamiento.

Como señala Pérez (2013a), las claves para esta adaptación requieren de diversos actores y elementos: profesorado, estudiantes, competencias y metodologías. Es bajo este marco que irrumpe de forma significativa la utilización de las TIC en el Espacio Europeo para la Educación Superior (Blayone, Mykhailenko, Van Oostveen y Barber, 2018; Cebreiro y Fernández, 2003; González, Román y Prendes, 2018; Nascimbeni y Burgos, 2018), pero también otras estrategias relevantes de innovación que comportan el favorecimiento de habilidades para la reflexión crítica del estudiante (Alonso y Arandia, 2014) y el reforzamiento de competencias para el trabajo en equipo, el trabajo en red o la implicación de la comunidad en la toma decisiones y en los procesos de evaluación (Lucas, García y Vega, 2015), así como en la incorporación del aprendizaje de lenguas extranjeras (Cavalcante, Riberas y Rosa, 2016), etc. Elementos todos que afectan de un mismo modo a las necesidades formativas del docente para su desarrollo, igual que a la mejora de las condiciones estructurales y/o organizativas y culturales que requiere este planteamiento.

En este contexto se presenta el objetivo del presente trabajo, con la intencionalidad de desarrollar una revisión sistemática de las innovaciones educativas introducidas en el contexto universitario del grado de Educación Social.

\section{Material y método}

Se considera que la metodología de la revisión sistemática es un procedimiento adecuado para señalar los estudios más significativos respecto de la innovación (Ramírez y García, 2018). La revisión de la literatura científica para esta investigación tomó como referencia las cinco etapas establecidas por Sánchez Meca y Botella (2010) para realizar estudios de revisiones sistemáticas y metaanálisis, y que han sido utilizadas con éxito dentro del campo de la educación por Monroy y Hernández Pina (2014). La primera de ellas fue la formulación 
del problema de investigación, para este caso se trataba de qué tipo de innovaciones se están llevando a cabo desde la implantación del EEES en el grado de Educación Social en España. Respecto a los criterios de selección, se incluyeron estudios con diseño, tanto cualitativos como cuantitativos y mixtos, redactados en español, inglés, catalán y portugués, realizados desde enero de 2007 hasta mayo de 2018 y que contenían datos, resultados y medidas concluyentes fruto de la inclusión de acciones de innovación educativa desarrollados específicamente en el grado de Educación Social. Se seleccionaron análisis sobre estudiantes y docentes universitarios de Educación Social desarrollados en España. Fueron excluidos los estudios teóricos, los ensayos narrativos o los que no tuvieran el foco de estudio principal en el grado de Educación Social.

Para la medida de la calidad de los análisis seleccionados se siguieron las directrices ofrecidas tanto por la guía de lectura crítica de Caspe para estudios cualitativos (Cabello, 2015), como por la escala PEDro para estudios cuantitativos (Sherrington, Herbert, Maher y Moseley, 2000). Ambas incluyen, entre otras, información acerca del tipo de diseño, selección de la muestra, estrategias de muestreo, descripción de las intervenciones y medidas de los resultados. Puntúan sobre un mínimo de 0 y un máximo de 10 puntos. Para aquellos estudios que emplearon una metodología mixta, se muestran los resultados de los indicadores obtenidos, tanto para el estudio cuantitativo como para el cualitativo. Para reforzar el indicador de la calidad de los artículos se obtuvo un segundo marcador, optándose por el ítem de la difusión internacional de la publicación (DI), que aglutina tanto el carácter selectivo de la revista en la que se publicó el artículo como la especialización de la temática a través del indicador facilitado por ANECA para tal fin (<http://epuc.cchs.csic.es/dice/ busqueda.php >). Se consideran una visualización baja las puntuaciones obtenidas por cada revista hasta 5 puntos; una visualización media, las puntuaciones entre 6 y 11 puntos, y una visualización alta, las puntuaciones superiores a 12 puntos. Para aquellos estudios en los que no se pudo localizar el indicador de difusión internacional (N/I), se realizó una búsqueda en la propia revista para comprobar si estaban indexadas en Latindex (c) o Scopus (s).

Respecto a las estrategias de búsqueda de los artículos, se consultó en bases de datos electrónicas (Google Scholar, ERIC, Scopus, Web of Science y Dialnet). Para su correcta identificación se emplearon los operadores booleanos Y/ AND y O/OR, combinando las siguientes palabras clave en el título y en el resumen de los artículos: (educational innovation) and (social education) or (degree of social education). Todo el proceso desarrollado se muestra a través de un diagrama de flujo en el gráfico 1.

Para la codificación de los estudios y la extracción de la información, una vez que se llevó a cabo la lectura del resumen, del título y de las palabras clave de todos los artículos, se seleccionaron aquellos que cumplían con los criterios de inclusión, siguiendo la estrategia PICOS, que recomiendan para el reporte de revisiones sistemáticas Rubio Aparicio, Sánchez Meca, Marín Martínez y López López (2018). Se elaboró el manual de características y protocolo de 
Gráfico 1. Diagrama de la búsqueda

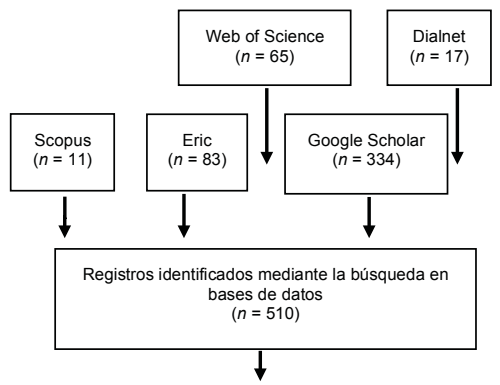

Registros eliminados tras leer el titulo, el resumen y los duplicados o que no cumplían con los criterios seleccionados de estudio primario
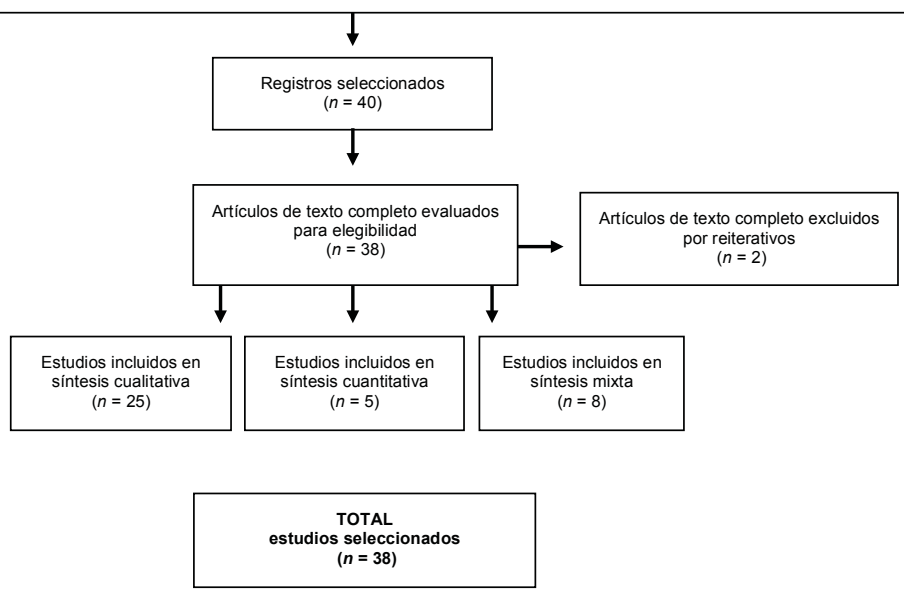

Fuente: elaboración propia.

registro para la extracción de los datos de los artículos. Se creó para ello una base de datos con las variables recogidas. Las variables que se extrajeron para cada uno de los estudios fueron: autor; título; comunidad autónoma y universidad; asignatura; tipo de innovación, que tomó como referencia la categorización y la clasificación realizada por García Peñalvo (2015); destinatarios; curso académico; duración; diseño de investigación; tamaño de la muestra; calidad del estudio; difusión de la publicación, y resultados, que fue categorizada como positiva o negativa, en función de la propia opinión expresada por los sujetos en los resultados de los estudios primarios seleccionados. Los análisis fueron codificados por dos investigadores, quienes volcaron los datos de manera independiente a la base de codificación de datos.

En cuanto al análisis de los artículos seleccionados, todos ellos fueron examinados en relación con los indicadores empleados para las variables seleccionadas. La información fue estudiada siguiendo un análisis cualitativo del contenido de los estudios apoyada por el cálculo de frecuencias y promedios. 


\section{Resultados y discusión}

Los resultados obtenidos se muestran en las tablas 1 y 2 .

Tabla 1. Características de la muestra

\begin{tabular}{|c|c|c|c|c|c|}
\hline \multicolumn{3}{|c|}{ PROCEDENCIA Y OBJETIVOS $(n=38)$} & \multicolumn{3}{|l|}{ DESTINATARIOS Y ASIGNATURAS $(n=38)$} \\
\hline & $n$ & $\%$ & & $n$ & $\%$ \\
\hline \multicolumn{3}{|c|}{ Contexto geográfico universitario } & \multicolumn{3}{|l|}{ Destinatarios } \\
\hline Andalucía & 19 & $50,0 \%$ & Estudiantes & 28 & $73,6 \%$ \\
\hline País Vasco & 6 & $15,7 \%$ & Comunidad educativa & 9 & $23,6 \%$ \\
\hline Región de Murcia & 6 & $15,7 \%$ & Profesionales & 1 & $2,6 \%$ \\
\hline Cataluña & 2 & $5,2 \%$ & \multicolumn{3}{|l|}{ Asignaturas implicadas } \\
\hline Comunidad Valenciana & 2 & $5,2 \%$ & $\mathrm{TIC}$ & 15 & $39,4 \%$ \\
\hline Castilla-La Mancha & 2 & $5,2 \%$ & Fundamentos Pedagógicos & 3 & $7,8 \%$ \\
\hline Castilla y León & 1 & $2,6 \%$ & Prácticum & 3 & $7,8 \%$ \\
\hline Galicia & 1 & $2,6 \%$ & Animación Sociocultural & 3 & $7,8 \%$ \\
\hline Comunidad de Madrid & 1 & $2,6 \%$ & Educación de Personas Adultas & 3 & $7,8 \%$ \\
\hline Objetivo de la innovación & & & Educación de Personas Mayores & 3 & $7,8 \%$ \\
\hline $\mathrm{TIC}$ & 10 & $26,3 \%$ & Didáctica General & 2 & $5,2 \%$ \\
\hline Portafolios & 7 & $18,4 \%$ & Planificación e Investigación & 2 & $5,2 \%$ \\
\hline Aprendizaje significativo & 5 & $13,1 \%$ & Propuestas Metodológicas Generales & 1 & $2,6 \%$ \\
\hline Metodología & 4 & $10,5 \%$ & Organización y Gestión & 1 & $2,6 \%$ \\
\hline Intergeneracional & 3 & $7,8 \%$ & Participación Social y Asociacionismo & 1 & $2,6 \%$ \\
\hline Infancia vulnerable & 1 & $2,6 \%$ & Medio Escolar & 1 & $2,6 \%$ \\
\hline Coordinación docente & 1 & $2,6 \%$ & Psicología Social y Organizaciones & 1 & $2,6 \%$ \\
\hline Mediación & 1 & $2,6 \%$ & Educación para el Desarrollo & 1 & $2,6 \%$ \\
\hline Creatividad & 1 & $2,6 \%$ & Evaluación de Proyectos y Servicios & 1 & $2,6 \%$ \\
\hline ABP & 1 & $2,6 \%$ & Mediación y Conflicto & 1 & $2,6 \%$ \\
\hline Responsabilidad social & 1 & $2,6 \%$ & Redes Sociales & 1 & $2,6 \%$ \\
\hline Aprendizaje-servicio & 1 & $2,6 \%$ & Intervención con Menores & 1 & $2,6 \%$ \\
\hline Prácticas & 1 & $2,6 \%$ & Todo el grado & 1 & $2,6 \%$ \\
\hline Animación sociocultural & 1 & $2,6 \%$ & & & \\
\hline
\end{tabular}

Fuente: elaboración propia. 


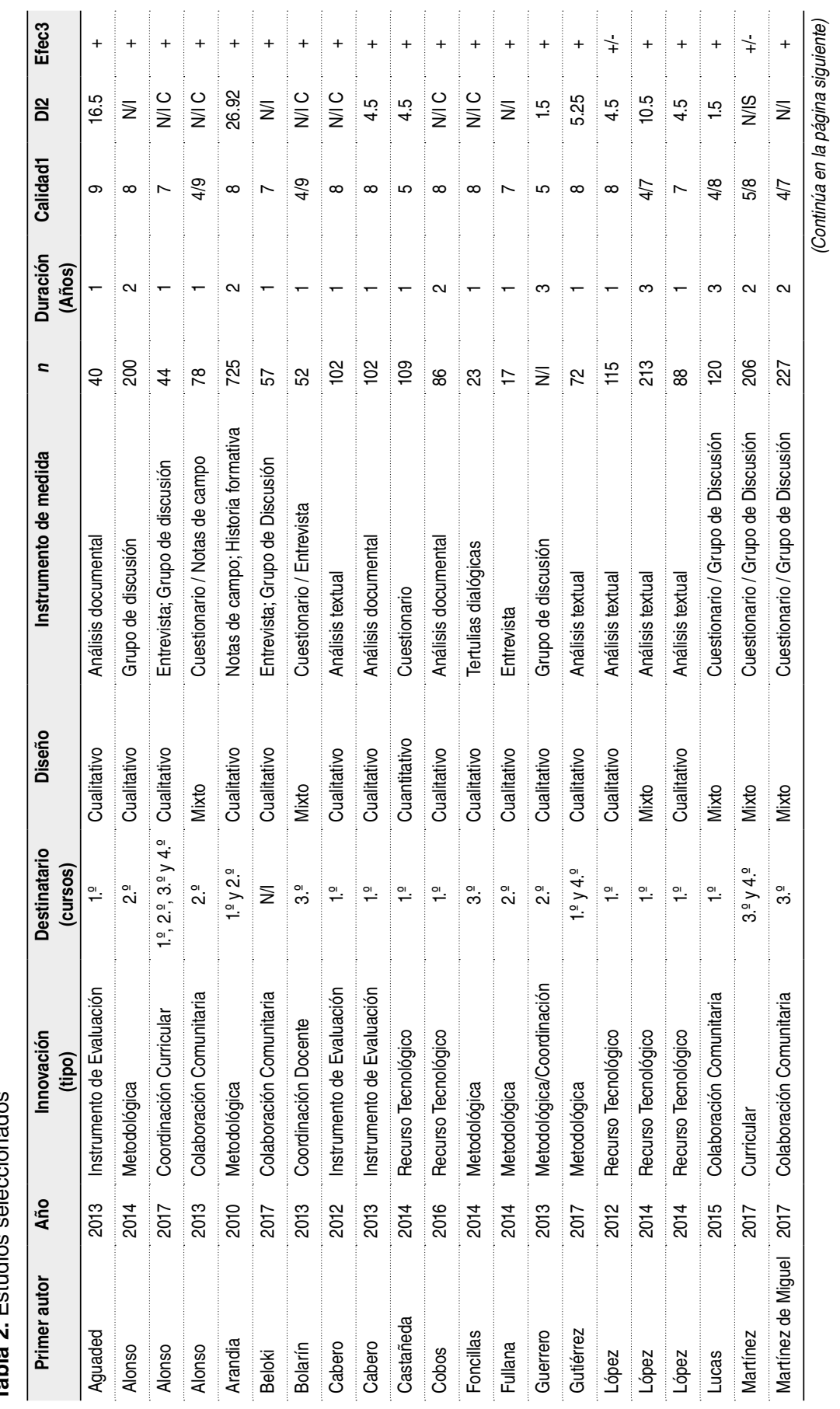




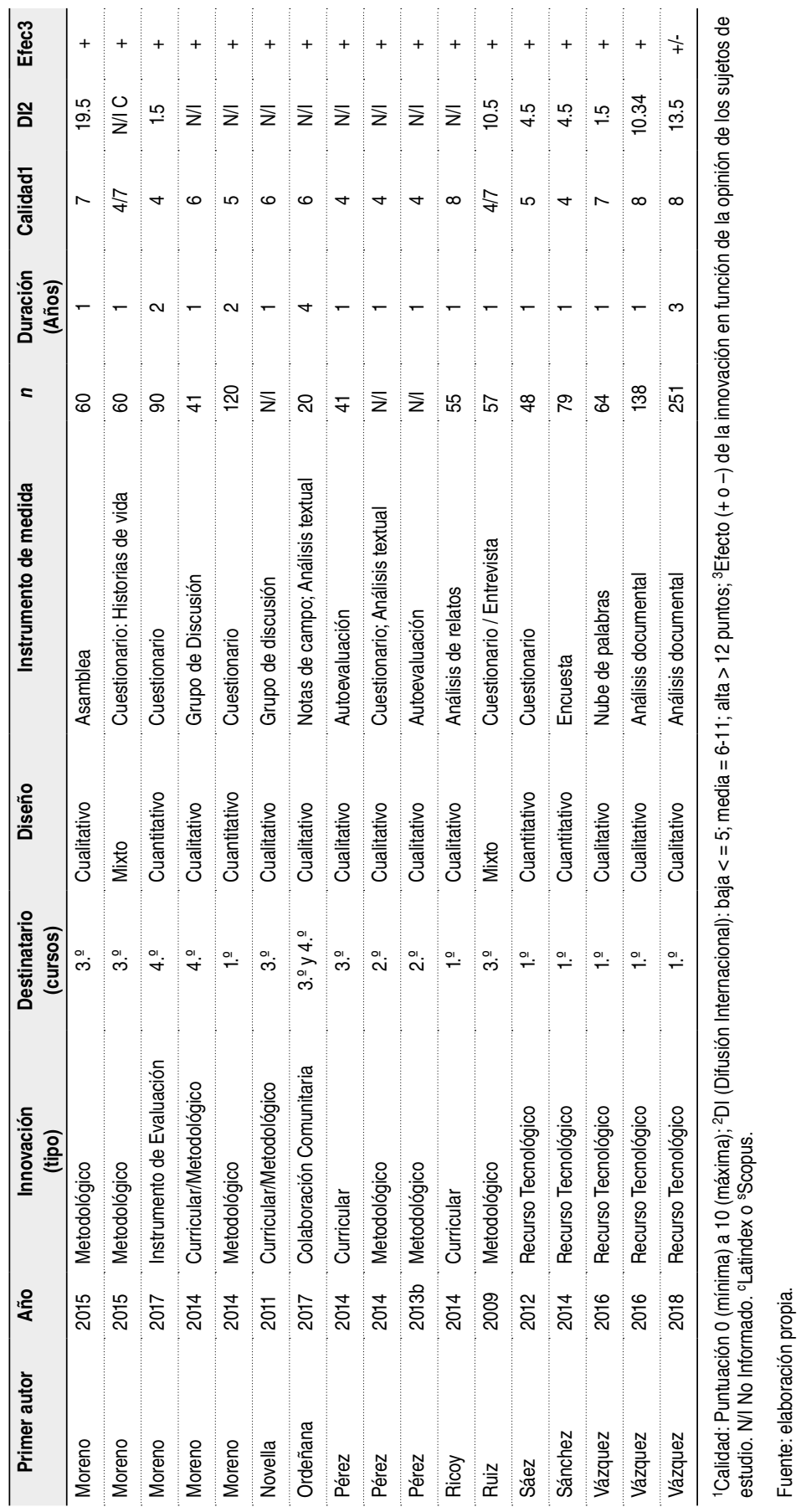


En coherencia con el objetivo de este trabajo - conocer la evolución de la innovación educativa en el grado de Educación Social en el contexto del Espacio Europeo de Educación Superior-, cabe señalar que los resultados muestran en primer lugar un incipiente, pero todavía escaso, desarrollo de investigaciones en este ámbito. De hecho, es en los últimos cinco años recogidos en el estudio donde se concentran la mayoría de los trabajos. De los 58 títulos de este grado que se imparten en España, solo se han hallado investigaciones relativas a la temática en un $22,41 \%(N=13)$ del total de universidades. De manera concreta, la mayor parte de estudios realizados sobre innovaciones educativas se agrupan principalmente en la Universidad Pablo Olavide de Sevilla, con un 47,36\% $(N=18)$, y en menor medida en la Universidad del País Vasco, con un 15,78\% $(N=6)$; en la Universidad de Murcia, con un $7,89 \%(N=3)$, y en la Universidad de Valladolid, con un 5,2\% $(N=2)$. El resto de instituciones de educación superior contemplan una única investigación al respecto. Dentro de los planes de estudio de este grado universitario, los resultados muestran que, respecto a la tipología y al predominio de las innovaciones realizadas, la mayor parte están referidas a aspectos metodológicos, con un $31,57 \%(N=12)$; a recursos tecnológicos, con un $26,31 \%(N=10)$, y en porcentajes menos significativos, a experiencias que tienen que ver con la colaboración comunitaria, un 13,15\% $(N=5)$; con aspectos curriculares, un $13,15 \%(N=5)$, con instrumentos de evaluación, un $10,52 \%(N=4)$, y con coordinación docente, un $5,26 \%(N=2)$. Si se profundiza en los objetivos que persiguen con la introducción de dichas innovaciones, el campo de las nuevas tecnologías constituye el grupo más numeroso. Precisamente, la principal asignatura en la que se han ido desarrollando ha sido la de Tecnologías de la Información y la Comunicación, con un 39,47\% $(N=15)$. A este respecto, coincide que el curso donde tienen lugar la mayor parte de esas innovaciones sea primero, dado que en el diseño del plan de estudios esta asignatura suele ubicarse precisamente en ese año de formación. El análisis realizado por Castañeda, Gutiérrez y Román (2014) concluye que un ambiente enriquecedor de experiencias tecnológicas puede favorecer estudiantes más independientes, autorregulados, capaces de manejar y enriquecer su entorno de aprendizaje. No obstante, y como apuntan Guerrero, Gutiérrez y Miquel (2018), es necesario imbricarlas en un modelo educativo contextualizado. En un porcentaje significativo destaca la incorporación del uso del portafolio en la formación universitaria, en el grado de Educación Social, con un 18,42\% de las experiencias analizadas. Aunque podría estar asociado al tipo de experiencias anteriores, existe un porcentaje de innovaciones (un 13,15\%) que refieren como objetivos el hecho de que el alumnado consiga un aprendizaje reflexivo, dialógico y significativo. También muestran los resultados un creciente interés por introducir innovaciones que tienen que ver con la apertura a la comunidad (un 13,15\%); relativos a la incorporación de proyectos intergeneracionales, de aprendizaje-servicio o protección a la infancia vulnerable, y por la adquisición de la competencia investigadora, con un $10,52 \%$ de las investigaciones realizadas. De manera testimonial, se encuentran estudios que 
se plantean como objetivos favorecer la coordinación docente, mejorar el prácticum, favorecer la responsabilidad social y desarrollar experiencias de animación sociocultural, así como trabajar las competencias en materia de mediación y creatividad.

En relación con el tipo de investigaciones realizadas sobre las innovaciones educativas introducidas, habría que destacar que, en materia de investigación en el grado de Educación Social de las universidades españolas, existe un predomino fundamental de optar por un diseño cualitativo, con un $65,78 \%$ $(N=25)$, de los cuales son minoritarios los que utilizan un software informático para el análisis de los datos. En menor medida se identifica la elección de un diseño mixto de investigación, con un $21,05 \%(N=8)$, y como opción menos seleccionada investigaciones estrictamente de carácter cuantitativo, como un $13,15 \%(N=5)$. Realizado el análisis de la calidad de la evidencia metodológica de los estudios seleccionados y teniendo en cuenta las variables y las escalas analizadas, cabe decir que los estudios presentan un valor medio $(\mathrm{M}=5,7 ; \mathrm{ST}=1,5)$, siendo la puntuación de los que emplean estrategias cualitativas algo superior, con una calidad promedio valorada como moderada $(\mathrm{M}=7,15 ; \mathrm{ST}=1,34)$ respecto a los estudios cuantitativos, cuya calidad de la evidencia metodológica presenta una valoración menor $(\mathrm{M}=4,3$; $\mathrm{ST}=0,43)$.

De la misma manera, si se atiende al índice de difusión internacional de los estudios, solo se pudo obtener para el $47 \%$ de los artículos seleccionados, de los cuales tan solo el $22 \%$ presenta una difusión internacional elevada. El estudio que presenta mayor puntuación en este sentido es el de Arandia, Alonso y Martínez (2010), que utiliza 725 informantes. Las investigaciones realizadas hasta el momento permiten afirmar la existencia de proyectos de innovación educativa preferente, aunque no exclusivamente, de un año académico de duración y dirigidos al alumnado en situación grupal, coincidiendo con los principios de la Declaración de Bolonia. En una proporción bastante menos significativa destacan aquellas innovaciones dirigidas a docentes, con un $15,78 \%$, y con el mismo porcentaje, experiencias que tienen que ver con la comunidad, los colectivos de profesionales, las entidades, las personas mayores o las familias.

Para finalizar, y recogiendo la valoración de las innovaciones por parte de los participantes, cabe destacar que los destinatarios en su totalidad dan una visión satisfactoria de la introducción de innovaciones educativas en su formación universitaria. En general, son mayoritarias las aportaciones que relatan sus beneficios y se podrían desglosar en función del favorecimiento de un aprendizaje autorregulado, significativo y participativo, con un $47,36 \%$. En segundo lugar, con un 26,31\%, los participantes hablan de la generación de un pensamiento crítico y de una meta de aprendizaje gracias a la introducción de dichas innovaciones. En un porcentaje también relevante refieren los beneficios para su proceso de enseñanza-aprendizaje en el aula, con un 21,05\%. De otro lado, los implicados manifiestan beneficios referidos a su profesionalización y a su desarrollo de trabajo en equipo y cooperativo. También son recurrentes las referencias a la responsabilidad social que desde la institución 
universitaria se tiene con la sociedad, expresándose en términos de mejora de la convivencia, de compromiso profesional o de empoderamiento social. Son muy pocas las excepciones que reflejan limitaciones en sus resultados, no obstante se señalan algunas oportunidades de mejora en referencia a las TIC (López, Fernández, Cobos y Pedrero, 2012; Vázquez, López, Fernández y Ballesteros, 2018).

\section{Conclusiones}

El propósito de este trabajo era hacer un recorrido por la investigación realizada en torno a la introducción de acciones de innovación educativa en el grado de Educación Social de las universidades españolas, para tratar de constatar si se había dado un cambio de filosofía en la concepción de la enseñanza universitaria desde la creación del Espacio Europeo de Educación Superior.

Aunque se constatan trabajos llevados a cabo desde el año 2007, no se puede afirmar que correspondan a un volumen amplio de los mismos. Quizá entre las razones principales se encuentren que no hay numerosos grupos de investigación que se estén dedicando a profundizar en esta línea de acción. Tal como se ha podido constatar en el análisis realizado, es un mismo tipo de profesionales los que suelen publicar experiencias en esta línea, a lo que se podría sumar la incorporación de innovaciones, aunque no su análisis ni su evaluación, puesto que, al establecer como criterio de selección el de la tipología de investigaciones, el número de referencias descendió notablemente. Muchos de los trabajos encontrados, y desechados por ello para esta revisión, se centraron en la descripción de la innovación educativa, en lugar de referirse a la investigación correspondiente a la misma.

Los datos han reflejado, además claramente, dos cuestiones principales relevantes en materia de análisis que pueden ofrecer nuevos retos a trabajar en el futuro. De un lado, el predominio de la investigación cualitativa a la hora de abordar este ámbito de un estudio, dadas las características de las investigaciones analizadas en cuanto a tiempos, espacios, participantes, instrumentos y procedimientos analíticos. Ello puede reflejar la necesidad de dar respuesta a la práctica cotidiana y concreta de los docentes en su aula, un aspecto que es muy relevante para establecer propuestas de mejora, pero quizá impide un escalamiento de variables, dimensiones y generalización de estrategias, lo que permitiría una extrapolación y una pormenorización en el proceso analítico de resultados o la tendencia a realizar un tipo de investigación que realice una combinación metodológica de instrumentos de obtención de información, con un mayor número de participantes, longitudinalidad y diversidad de recursos de acceso a la información. De otro lado, y relacionado con ello, estaría la mejorable visibilidad y difusión que tiene el trabajo que se está realizando hasta ahora en la comunidad científica, por el predominio de publicaciones en revistas de menor impacto en las que se han dado a conocer las investigaciones realizadas, con las implicaciones que esta situación conlleva, lo que, como puede percibirse del análisis elaborado, conduce al desconocimien- 
to de las innovaciones de calidad que se están realizando. Por dicho motivo sería importante llevar a cabo una apuesta en el ámbito científico y que hubiera un interés creciente hacia este contexto de investigación como es la formación universitaria.

No obstante, a pesar de los condicionantes encontrados, se constata el número considerable de beneficios que conlleva la introducción de proyectos de estas características en las aulas universitarias. La mayor parte de investigaciones analizadas ponen de manifiesto las nuevas competencias que adquieren los agentes implicados: de un lado, se ha realizado una apertura al exterior frente a planteamientos que contemplaban la universidad como un ente aislado de la realidad social, ofreciendo nuevas posibilidades al ámbito de las nuevas tecnologías como herramientas cotidianas de trabajo, al igual que en el resto de los contextos de la sociedad. En este estudio se destaca que las TIC han irrumpido con fuerza, como se ha podido comprobar en la formación universitaria, lo que ha constituido un elemento favorecedor de adaptación a las exigencias de las demandas sociales, así como una nueva redefinición de sus relaciones con la comunidad para trabajar de manera más coordinada y acorde a las necesidades reales que tienen los ciudadanos destinatarios de la acción social de la Educación Social como derecho adquirido. Del mismo modo, la innovación ha posibilitado un cambio sustancial en las competencias a adquirir, tanto por parte de los estudiantes como de los docentes, al propiciar una nueva conceptualización en torno a la autonomía, al compromiso y a la responsabilidad de los agentes intervinientes en los procesos de formación universitaria. Prueba de ello es el éxito constatado de la introducción de metodologías e instrumentos que promueven la implicación y el protagonismo del alumnado, ofreciéndole herramientas para generar recursos que conducen a un proceso de enseñanza-aprendizaje más autónomo, pero al mismo tiempo más tendente a la cooperación, más reflexivo y más conducente a facilitar su futura profesionalización. La relevancia que adquiere la mayor implantación y evaluación por parte del profesorado de proyectos de innovación educativa en educación social conllevaría un beneficio tendente a la mejora en los procesos de aprendizaje y competitividad, toda vez que el paradigma tradicional de la mera transmisión de conocimientos está caduco.

Como limitación de la investigación presentada, cabe señalar la dificultad encontrada a la hora de poder seleccionar y obtener estudios primarios u originales con los criterios de selección propuestos, por lo que poder obtener unidades de análisis podría plantearse como una importante limitación en el campo de la investigación en materia de docencia universitaria. En otras palabras, se está produciendo un número mayor de experiencias puestas en práctica de proyectos de este tipo cotidianamente en muchas universidades españolas, pero no han ido acompañadas del desarrollo de un estudio primario correspondiente.

Por lo que, del análisis realizado, se puede concluir que la apuesta que introducía este cambio paradigmático refleja resultados esperanzadores y optimistas. Es importante incorporar este tipo de propuestas educativas con con- 
tinuidad e integración curricular en la docencia universitaria. Quizá haya que trabajar en la línea de mayores investigaciones y desempeñarse con mayor rigurosidad en torno a este respecto desde la comunidad científica.

\section{Referencias bibliográficas}

Aguaded, J.C.; LÓpez, E. y JaÉN, A. (2013). Portafolios electrónicos universitarios para una nueva metodología de enseñanza superior: Desarrollo de un material educativo multimedia. Revista de Universidad y Sociedad del Conocimiento (RUSC), 10(1), 7-28. <http://dx.doi.org/10.7238/rusc.v10i1.1333>

Alonso, I. y Arandia, M. (2014). Aprender creando: Factoría creativa en las aulas universitarias. Revista de Docencia Universitaria, 12(1), 443-468. $<$ https://doi.org/10.4995/redu.2014.6419>

Alonso, I.; Arandia, M.; Martínez, I.; Martínez, B. y Gezuraga, M. (2013). El Aprendizaje-Servicio en la innovación universitaria: Una experiencia realizada en la formación de educadoras y educadores sociales. Revista Internacional de Educación para la Justicia Social, 2(2), 195-216.

Alonso, I.; Artexe, K.; Berasategui, N. y Arandia, M. (2017). Avanzando hacia una cultura docente y curriculum interdisciplinar, colaborativo y conectado con la realidad profesional: La innovación curricular en el Grado de Educación Social (UPV/EHU). IV Congreso Internacional sobre Aprendizaje, Innovación y Competitividad (CINAIC 2017). Zaragoza, del 4 al 6 de octubre.

Arandia, M.; Alonso, M.J. y Martínez, I. (2010). La metodología dialógica en las aulas universitarias. Revista de Educación, 352, 309-329.

Beloki, N.; Martínez, I.; Remiro, A. y Zarandona, E. (2017). Buenas Prácticas en Educación Social con infancia en situación de desprotección: Claves para la intervención desde las voces de personas participantes y profesionales. Revista de Educación Social, 24, 1188-1205.

Blayone, T.J.B.; Mykhailenko, O.; Van Oostveen, R. y Barber, W. (2018). Ready for digital Learning?: A mixed-methods exploration of serveyed technology compettencies and authentic performance activity. Education and Information Technologies, 23(3), 1377-1402.

Bolarín, M.J.; Moreno, M.A. y Porto, M. (2013). Coordinación docente e interdisciplinariedad: Análisis de su contribución a la adquisición de competencias docentes y discentes. Revista de Docencia Universitaria, 11(2), 443-462.

Cabello, J. B. (2015). Lectura critica de la evidencia clínica. Barcelona: Elsevier.

Cabero, J.; López, E. y JaÉn, A. (2013). Los portafolios educativos virtuales en las aulas universitarias: Instrumentos didácticos para la innovación docente y la calidad de los procesos de enseñanza y aprendizaje. Enseñanza \& Teaching, 31, 43-70.

Cabero, J.; López, E. y Llorente, M.C. (2012). E-Portafolio universitario como instrumento didáctico 2.0 para la reflexión, evaluación e investigación de la práctica educativa en el espacio europeo de educación superior. Virtualidad, Educación $y$ Ciencia, 3(4), 27-45.

Castañeda, L.; Gutiérrez, I. y Román, M.M. (2014). Enriqueciendo la realidad: Realidad aumentada con estudiantes de Educación Social. Revista de Innovación Educativa, 12, 15-25. $<$ https://doi.org/10.7203/attic.12.3544> 
Cavalcante, M.T.L.; Riberas, G. y Rosa, G. (2016). Fostering innovation in social work and social education degrees: Multilingual environment and tools for social change. International Journal of Educational Technology in Higher Education, 13, 31. <https://doi.org/10.1186/s41239-016-0031-0>

Cebreiro, B. y Fernández, C. (2003). Las tecnologías de la comunicación en el espacio europeo para la educación superior. [The technologies of the education in the European space for the university education.] Comunicar, 21, 57-61.

Cobos, D.; López, E. y Llorent, M. (2016). Propuesta de Innovación Didáctica con Portafolios Electrónicos en Formato Blog: Un Caso de Estudio en una Universidad. Formación Universitaria, 9(6), 27-42. <http://dx.doi.org/10.4067/S0718-50062016000600004>

Delgado, A. y Justo, E. de (2018). Evaluación del diseño, proceso y resultados de una asignatura técnica con aprendizaje basado en problemas. Educación XXI, 21(2), 179-203. <https://doi.org/10.5944/educxx1.19415>

DíAZ, F. (2010) Los profesores ante las innovaciones curriculares. Revista Iberoamericana de Educación Superior, 1(1), 1-10.

Foncillas, M. y Laorden, C. (2014). Tertulias Dialógicas en Educación Social: Transformando el Aprendizaje. International Journal of Sociology of Education, 3(3), 244-268. <http://dx.doi.org/10.4471/rise.2014.16>

Fullana, J.; Pallisera, M.; Palaudarias, J.M. y Badosa, M. (2014). El desarrollo personal y profesional mediante el aprendizaje reflexivo: Una experiencia en el grado de Educación Social. Revista de Docencia Universitaria, 12(1), 373-397.

García Peñalvo, F.J. (2015). Mapa de Tendencias e Innovación Educativa. Education in the Knowledge, 16(4), 6-23. <http://dx.doi.org/10.14201/eks2015164623>

González, V.; Román, M.M. y Prendes, M.P. (2018). Análisis de la mejora de la competencia digital del alumnado universitario a través del trabajo por tareas. En C. Guerrero y J.A. López (eds.). Innovación, diversidad y TIC en la enseñanza superior (pp. 352-358). Murcia: Editum.

Guerrero, E. y Calero, J. (2013). El aprendizaje basado en proyectos como base metodológica en el grado de Educación Social. Educación Social: Revista de Intervención Socioeducativa, 53, 73-91.

Guerrero, C.; Gutiérrez, M. y Miquel, A. (2018). Estrategias metodológicas interactivas en la formación de graduados en educación social. En C. Guerrero y P. Miralles (eds.). Innovación y modelos de enseñanza-aprendizaje en la educación superior (pp. 121-130). Murcia: Editum.

Gutiérrez, J.M.; Fernández, E. e Iglesia, L. de la (2017). Narrativas transmedia con jóvenes universitarios: Una etnografía digital en la sociedad hiperconectada. Anàlisi: Quaderns de Comunicació i Cultura, 57, 81-95. $<$ https://doi.org/10.5565/rev/analisi.3108>

López, E.; Fernández, R.; Cobos, D. y Pedrero, E. (2012). Implicaciones de las Tics en el ámbito socio-educativo y de servicios sociales: Una experiencia universitaria de innovación y desarrollo docente con tecnologías 2.0. Campo Abierto, 31(2), 11-35.

López, E.; Llorent, V. y FernÁndez, E. (2014). Experiencias universitarias en diferentes titulaciones de la Universidad Pablo de Olavide: Construcción de ideas 
previas sobre la sociedad de la información con software social 2.0. Didáctica, Innovación y Multimedia, 23, 1-12.

López, E.; VÁzquez, E. y Fernández, E. (2014). Análisis de la percepción de los estudiantes sobre las áreas de intervención del futuro educador y trabajador social a través de una didáctica digital con mapas conceptuales multimedia. RED: Revista de Educación a Distancia, 41, 150-166.

Lucas, S.; García, M.A. y Vega, C. (2015). Promoción de actitudes e iniciativas intergeneracionales: Responsabilidad social universitaria, orientación vocacional y aprendizaje-servicio. Revista Pedagógica, 28, 215-234.

Martínez, M.J.; Lloret, C. y Mas, S. (2017). Responsabilidad Social Universitaria (RSU): Principios para una universidad sostenible, cooperativa y democrática desde el diagnóstico participativo de su alumnado. Archivos Analíticos de Políticas Educativas, 25(75), 1-21. <https://doi.org/10.14507/epaa.25.2769>

Martínez de Miguel, S. (2007). Una experiencia de innovación del portafolio del alumno, en la Diplomatura de Educación Social, desde el marco de la Educación Superior en Europa. Educatio Siglo XXI, 25, 125-144.

Martínez de Miguel, S.; Salmerón, J.A. y Escarbajal de Haro, A. (2017). El proyecto intergeneracional universitario (Esium Proyect). En S. MARTínez DE Miguel, P. Moreno Abellán y A. Escarbajal de Haro. Envejecimiento Activo, Programas Intergeneracionales y Educación Social (pp. 147-174). Madrid: Dykinson.

Matas, A.; Tójar, J.C. y Serrano, J. (2004). Innovación educativa: Un estudio de los cambios diferenciales entre el profesorado de la Universidad de Málaga, Redie, 6(1), 1-21.

Michavila, F. (2009). La innovación educativa: Oportunidades y barreras. ARBOR: Ciencia, Pensamiento y Cultura, 185, 3-8.

Monroy, F. y Hernández Pina, F. (2014). Factores que influyen en los enfoques de aprendizaje universitario: Una revisión sistemática. [Factors affecting student approaches to learning: A systematic review.] Educación XX1, 17(2), 105-124. $<$ https://doi.org/10.5944/educxx1.17.2.11481>

Moreno, O. y Martín, N. (2014). Aproximación a la historia de vida a partir de un taller de formación con el alumnado de $1^{\circ}$ de Grado de Educación Social. Reire, 7(2), 91-104. <http://dx.doi.org/10.1344/reire2014.7.2726>

Moreno, O. y Moreno, P. (2015a). Jóvenes investigadores en las aulas universitarias: Una experiencia de innovación docente en el Grado de Educación Social. Revista de Docencia Universitaria, 13(2), 345-361.

- (2015b). De la pregunta de investigación a la publicación científica: Innovación educativa con el alumnado universitario. Cultura y Educación, 27(4), 899-928. <https://doi.org/10.1080/11356405.2015.1089386>

- (2017). El portafolio digital como herramienta didáctica: Una evaluación crítica de fortalezas y debilidades. Revista de Humanidades, 30, 11-30. <https://doi.org/10.5944/rdh.30.2017.18200>

Moreno, P. y PÉREZ, I. (2014). Estereotipos sobre la jubilación en pretitulados universitarios: Proyecto de innovación docente. Reire, 7(2), 53-70. <https://doi.org/10.1344/reire2014.7.2724>

Nascimbeni, F. y Burgos, D. (2018). Nuevas alfabetizaciones digitales para docentes en sociedades abiertas y colaborativas. En C. Guerrero y J.A. López (eds.). Innovación, diversidad y TIC en la enseñanza superior (pp. 401-420). Murcia: Editum. 
Novella, A.M.; Fuentes, N.; Forés, A. (2011). El disseny d'uns quaderns de Pràcticum des de la millora docent i el treball coresponsable per a la transferència de competències i el desenvolupament profesional. Reire, 4(2), 40-58. <http://dx.doi.org/10.1344/reire2011.4.2424>

Ordeñana, M.B.; Darretxe, L. y Beloki, N. (2017). Innovando el Practicum en educación social: Experiencia de trabajo colaborativo a partir de sus protagonistas. Revista Iberoamericana de Educación Superior, 7(20), 114-134.

Pablos, J. de (2007). El cambio metodológico en el Espacio Europeo de Educación Superior y el papel de las tecnologías de la información y la comunicación. Ried, $10(2), 15-44$. $<$ https://doi.org/10.5944/ried.2.10.992>

PÉREZ, I. (2013a). Innovación docente en animación sociocultural: Un modelo basado en los principios del espacio europeo de educación superior. Innovación Educativa, 23, 197-213.

- (2013b). Una experiencia de innovación docente en la asignatura de Animación Sociocultural. Reire, 6(2), 132-149.

- (2014). El trabajo en equipo mediante el uso del portafolio y las rúbricas de evaluación: Innovación en la enseñanza universitaria. Reire, 7(1), 56-75.

Pérez, I.; Moral Arroyo, G. del y Povedano Díaz, M.A. (2014). La mediación en Educación Social a través de una metodología participativa: Construcción colaborativa del conocimiento. Revista de Formación e Innovación Educativa Universitaria, 7(3), 117-129.

Ramírez, M.S. y García, F.J. (2018). Co-creación e innovación abierta: Revisión sistemática de la literatura. [Co-creation and open innovation: Systematic literature review.] Comunicar, 54, 9-18. <https://doi.org/10.3916/C54-2018-01>

Ricoy, M.C. y Couto, M.J. (2014). As boas práticas com TIC e a utilidade atribuída pelos alunos recém integrados à universidade. Educaçao e Pesquisa, 40(4), 897-912. <http://dx.doi.org/10.1590/S1517-97022014005000005>

Rubio, M.; Sánchez Meca, J.; Marín Martínez, F. y López López, J.A. (2018). Recomendaciones para el Reporte de Revisiones Sistemáticas y Meta-análisis. Anales de Psicología, 34(2), 412-420. $<$ http://dx.doi.org/10.6018/analesps.34.2.320131>

Ruiz, C. (2009). Hacia el aprendizaje significativo y colaborativo: Una propuesta de innovación en la diplomatura de Educación Social. Innovación Educativa, 19, 263-273.

SázZ, J.M. (2012). Integración práctica de la tecnología educativa en el grado de educación social. Revista Electrónica de Tecnología Educativa, 40, 1-16. <https://doi.org/10.21556/edutec.2012.40.369>

SÁnchez, J. y Morales, S. (2014). Desarrollando competencias de investigación a través de metodologías activas en un entorno B-learning. Contextos Educativos, 17, 23-44, <http://dx.doi.org/10.18172/con.2591>

Sánchez Meca, J. y Botella, J. (2010). Revisiones sistemáticas y meta-análisis: Herramientas para la práctica profesional. Papeles del Psicólogo, 31(1), 7-17.

Sherrington, C.; Herbert, R.D.; Maher, C.G. y Moseley, A.M. (2000). PEDro: A database of randomized trials and systematic reviews in physiotherapy. Manual Therapy, 5(4), 223-226. <http://dx.doi.org/ 10.1054/math.2000.0372> 
Sirignano, F.M. (2017). El modelo europeo de Universidad y los retos pedagógicos en la sociedad globalizada. En R. RoIG-VILA (ed.). Investigación en docencia universitaria: Diseñando el futuro a partir de la innovación educativa (pp. 3-7). Barcelona: Octaedro.

VÁzQuEz, E. y López, E. (2016). Experiencia didáctica con mapas conceptuales interactivos con estudiantes universitarios sobre las áreas de intervención socio-laboral del educador social. Aula de Encuentro, 18(1), 5-23.

VÁzquez, E.; López, E. y Fernández, E. (2016). Análisis diacrónico de la percepción del estudiantado sobre la Sociedad de la Información con software social. Didáctica, Innovación y Multimedia, 34, 1-11.

VÁzquez, E.; López, E.; Fernández, E. y Ballesteros, C. (2018). Los nuevos entornos virtuales de aprendizaje permanente (MOOC) y sus posibilidades educativas en ámbitos sociales y educativos. Revista de Medios y Educación, 53, 9-24.

<https://doi.org/10.12795/pixelbit.2018.i53.12> 